\title{
Posterior Endodontic Surgery - A Case Report
}

\author{
Tsvetelina Borisova-Papancheva ${ }^{1}$, Georgi \\ Papanchev $^{2}$, Stefan Peev ${ }^{3}$, Tihomir Georgiev ${ }^{2}$
}

1. Department of Conservative Dentistry and Oral Pathology,FDM, Medical University of Varna;

2. Department of Oral and Maxillofacial Surgery, FDM, Medical University of Varna;

3. Department of Periodontology and Dental Implantology, FDM, Medical University of Varna;

\begin{abstract}
Objective: Endodontic surgery is a standard procedure which enhances the retention* survival rate of many teeth which can not be treated only endodontically (inaccessibility to the apical part of the root by a coronal approach due to posts, root fractures, separated instruments in root canals, calcified and tortuous root canals, dens in dente, , etc.). Endodontic surgery can be applied both to the anterior and posterior regions on the maxilla and mandibula. In posterior regions the surgical procedure is more difficult due to anatomical conditions (maxillary sinus, mandibular canal, number of root canals, inaccessible posterior oral cavity, etc).

Aim: To present a difficult clinical case where endodontic surgery was chosen as the most - convenient method for treatment because by the routine endodontic treatment is not possible to remove a separated instrument in the periapical lesion.

Methods: The endodontic treatment was performed on tooth \#36, followed by endodontic obturation of the root canal system. In two days an apicoectomy without retrograde filling was performed.

Results: One year after the apicoectomy there is complete healing of the periapical lesion with new bone formation and no symptoms in the patient.
\end{abstract}

Keywords: apicoectomy, endodontic surgery, posterior region 


\section{Introduction}

Non-surgical endodontic management of periapical lesions is a routine predictable procedure for treatment because of its high success rate (1). However, there are valid indications for endodontic surgery (2). When it is indicated in the anterior region it is usually done without hesitation, but if it is necessary to perform it in the posterior region, endodontic surgery is often avoided and replaced by tooth extraction and implantation or intentional replantation of mandibular premolars and molars (3). The reasons to bypass endodontic surgery in the posterior region may be anatomical structures such as the maxillary sinus, the mandibular canal, limited access in this part of the oral cavity and lack of operator experience, $(4,5,6)$. When endodontic surgery is the only choice to keep the tooth it should not be avoided. However, it is necessary to have full knowledge of the surgical technique and the anatomical structures and variations $(6,7)$. Cone-beam computed tomography helps to predict the results and avoid complications (5). Most of the authors recommend performing a root-end cavity filling after apicoectomy $(8,9,10)$. The root-end filling avoids the microleakage but additional skills are also necessary, time and resources to prepare retrograde cavity and to make a retrofill should also be taken into consideration.

\section{Case Report}

A 22-year-old female patient was referred to the Department of Conservative Dentistry, FDM, Medical University-Varna by a general dentist for retreatment of tooth \#36. He found the presence of a periapical lesion between the roots of the tooth \#36 and a separated file in this lesion. An anamnesis was taken and clinical examination conducted, as well as a radiographic examination which included a periapical radiograph of tooth \#36 and a CBCT of the jaws (fig.1).

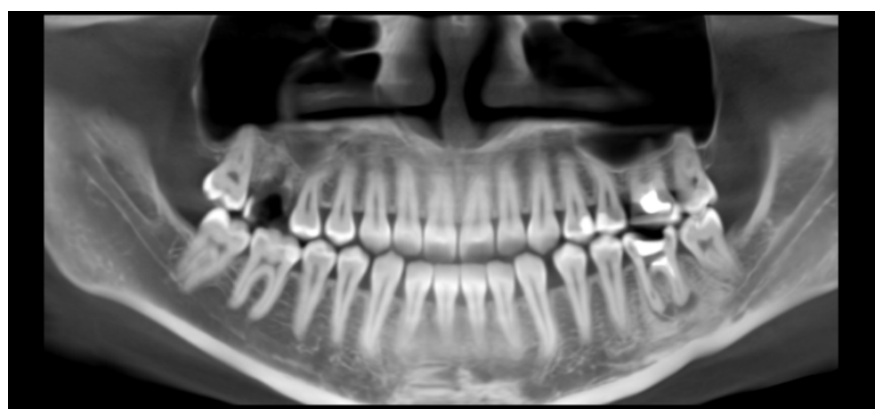

Fig.1. Initial CBCT

The periapical radiograph revealed radiolucency involving enamel, dentine, cementum and pulp in relation to tooth \#36 with a separated file in the cavity (fig.2). A well circumscribed periapical radiolucency of about $2 \mathrm{~cm}$ in diameter, involving both the mesial and distal roots of \#36, was registered. An endodontic access preparation on tooth \#36 was made without anaesthesia. The necrotic pulp tissue was extirpated followed by copious irrigation with sodium hypochlorite $5,25 \%$, citric acid $40 \%$ and normal saline. Working length was determined by using an apex-locator NSK and the root canals were prepared using machine files Protaper Next X2 (Dentsply Maillefer, Switzerland). The root canals were filled with a sealer Topseal (Dentsply Maillefer, Switzerland) by applying the method of warm vertical condensation (fig. 3). 
The patient was referred to the Department of Oral and Maxillofacial Surgery, FDM, Medical UniversityVarna for apicoectomy of the roots of tooth \#36 after 48 hour.

Under local anesthesia access was gained for tooth \#36. The triangular muco-periostal flap was opened under local anesthesia. All necrotic bone was curetted via bone curette until the bone become clean, hard and healthy. Apices of the molar were resected and no retrograde cavity was prepared and no root-end filling was used. All debris were irrigated with normal saline until the surgical field became clean. The flap was sutured with a resorpable Vicryl $4 / O$ suture. The patient was recalled for post-operative checkups after 3 days (fig. 4), one week, 2 weeks and 1 year (fig. 5). No complaints were reported by the patient. The healing was complete without any scar. The histopathological report confirmed the lesion to be a radicular cyst.

After 6 months, through periapical radiographs, significant regression of lesions with new bone formation and no symptoms in the patient were observed (fig. 3).

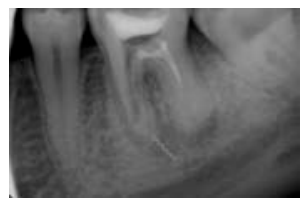

Fig.2. Initial periapical radiograph

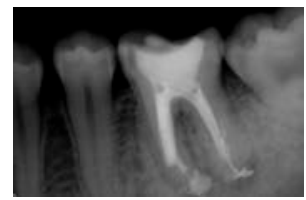

Fig. 3. A periapical radiograph showing an endodontic obturation of the root canal system 


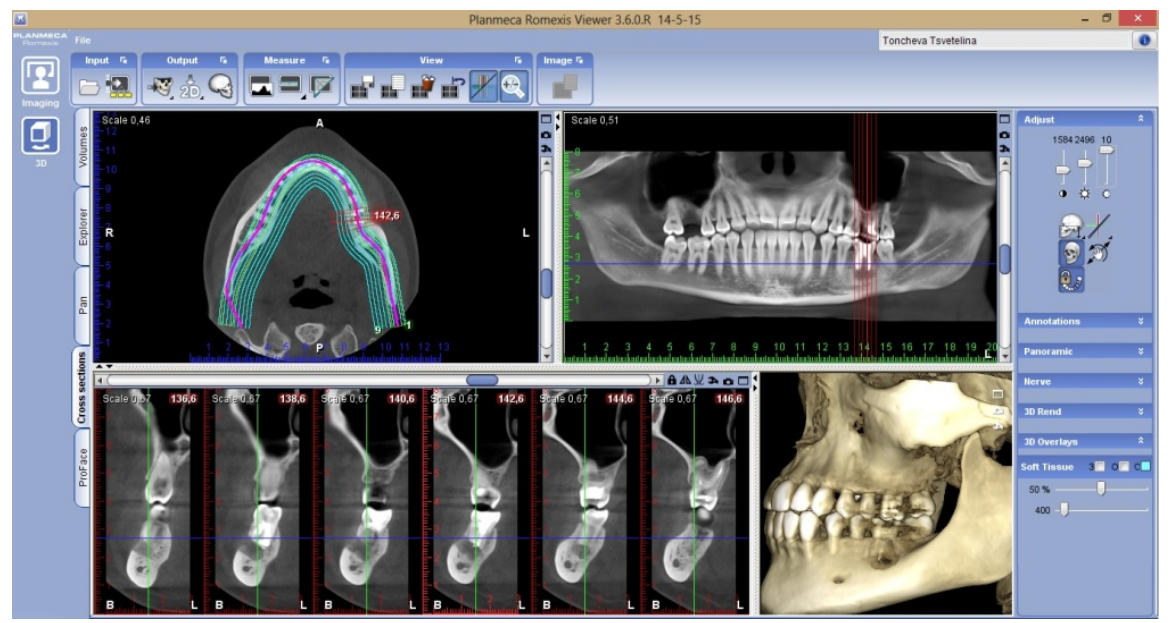

Fig. 4. СВСТ 3 days after the apicoectomy

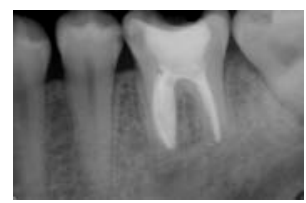

Fig. 5 A periapical radiograph after 1 year

\section{Results}

One year after the endodontic treatment and apicoectomy the radiograph (fig.5) shows significant recovery of the periapical lesion with new bone formation and no symptoms in the patient.

\section{Discussion}

This article shows that not every clinical case with apicoectomy needs to be performed with retrograde cavity and a root-end filling. The proper removal of irritants from the root canal system and its total obturation result in repair of inflamed periradicular tissues after apicoectomy (11). This case also demonstrates that apicoectomy is not only limited to the anterior region, but also can be performed on posterior teeth (7). Before doing it in the posterior region it is necessary for the oral surgeon to have a complete understanding of the anatomy and surgical techniques.

\section{Conclusion}

Apical periodontitis due to a periapically infected cyst or a chronic abscess can be treated by conventional root canal therapy when the lesion is small. Both surgical and nonsurgical combinations are indicated in teeth which cannot be treated endodontically (with broken instruments which cannot be removed under magnification). This case reports complete healing and new bone formation after apicoectomy of a tooth 
without any retrograde fillings. Thus the operation time, the risk of post-operative trauma as well as the cost of the manipulation were simultaneously reduced.

\section{References}

1. Borisova-Papancheva T., Panov Vl., Papanchev G., Peev S. Conservative non-surgical management of an extensive periapical lesion - a case report. Medlnform 2015; 2 (4): 364-369.

2. Gutman JL, Hovland EJ. A critical reappraisal of the routine use of periradicular surgery in conjunction with endodontics. J Dist Columbia Dent Soc. 1978, Summer, 53: 17-21.

3. Kingsbury, B. C., Wiesenbaugh, J. M. Intentional replantation of mandibular premolars and molars. The Journal of the American Dental Association, 1971, 83(5):1053-1057.

4. Grossman, L. I. Intentional replantation of teeth: a clinical evaluation. The Journal of the American Dental Association, 1982, 104 (5): 633-639.

5. Kalender, A., Aksoy, U., Basmaci, F., Orhan, K., Orhan, A. I. Cone-beam computed tomography analysis of the vestibular surgical pathway to the palatine root of the maxillary first molar. European journal of dentistry, 2013; 7 (1): 35.

6. Kim, S. G. Variations in Outcome of Endodontic Surgery. In Complications in Endodontic Surgery, 2014, Springer Berlin Heidelberg, 39-51.

7. Persson, G. Periapical surgery of molars. International journal of oral surgery, 1982, 11 (2): 96100.

8. Torabinejad, M., Higa, R. K., McKendry, D. J., Ford, T. R. P. Dye leakage of four root end filling materials: effects of blood contamination. Journal of Endodontics, 1994, 20 (4): 159-163.

9. Fischer, E. J., Arens, D. E., Miller, C. H. Bacterial leakage of mineral trioxide aggregate as compared with zinc-free amalgam, intermediate restorative material, and Super-EBA as a rootend filling material. Journal of endodontics, 1998, 24 (3): 176-179.

10. Borisova-Papancheva T, VI Panov, S Peev, G Papanchev. Root-end filling materials-review. Scripta Scientifica Medicinae Dentalis 2015, 1 (1): 9-15.

11. Green TL, et al. Radiographic and histologic periapical findings of root canal treated teeth in cadaver. Oral Surg 1997; 83: 707.

\section{Corresponding author:}

Tsvetelina Borisova-Papancheva

Faculty of Dental Medicine, Department of Conservative Dentistry and Oral Pathology,

Medical University -Varna

Email: dr_borisova@abv.bg 